\title{
Robert Stockhammer
}

Wie deutsch ist es?

\section{Glottamimetische, -diegetische, -pithanone, und -aporetische Verfahren in der Literatur}

DOI 10.1515/arcadia-2015-0009

\begin{abstract}
Recent research concerning the linguality of literature has yielded the insight that multilingual texts are in fact the rule, whereas monolinguality (in the pre-Derridean sense of the word: homogenously English, purely German, etc.) is the exception. Until now, however, no classification of different types of mutlilinguality has been established. In the present article, I propose such a classification and test it, primarily by using a sample of narrative texts by W. G. Sebald, Primo Levi, Anita Desai, Roberto Bolaño, Walter Abish, and James Joyce. Each of these texts, in one way or another, includes German elements even though only the first of these is usually regarded as pertaining to German literature. Borrowing terminological items from Plato, Aristotle, and Charles S. Peirce, I propose to distinguish glotta-mimesis (presence of other languages on the level of the discours) from glotta-diegesis (presence of other languages on the level of the histoire), to add a sub-distinction to the former class (iconic vs. indexical glotta-mimesis), and to introduce two further terms (glotta-pithanon and glottaaporetic) that will be useful in describing key aspects of the relationship between languages on the level of the discours and those on the level of the histoire. There are, however, cases in which it is impossible to determine the language spoken within the fictional ,world', as constructed by a particular literary text. This class of glotta-aporetic phenomena turns out to be more than just one easily definable class. Limiting the reach of any classification based on the seemingly discrete distinction between histoire and discours, this linguistic aporia marks the autonomy of literature.
\end{abstract}

Keywords: multilinguality, German literature, narratology

Prof. Dr. Robert Stockhammer: Allgemeine und Vergleichende Literaturwissenschaft, LudwigMaximilians-Universität, Schellingstr. 3, 80799 München, E-Mail: robert.stockhammer@lmu.de 


\section{Ein Kategorieninventar zur Beschreibung von Formen der Mehrsprachigkeit}

In literarischen Texten ist sehr häufig mehr als ein Idiom im Spiel. Dies gilt keineswegs nur von Texten, die gewöhnlich der postkolonialen oder MigrantenLiteratur zugeordnet werden, sondern, wie schon ein flüchtiger Blick zeigt, durchaus auch von älteren Texten, die einige kanonische Geltung besitzen: Die Protagonisten von Heliodors Aithiopika schlagen sich mit rudimentärem Ägyptisch durch; in Dantes Purgatorio wird gelegentlich Provenzalisch gedichtet; vom Don Quixote ist nur eine aus dem Arabischen übersetzte Fassung überliefert; auch von Mignons Italien-Lied kennt man nur Wilhelm Meisters Übersetzung. Das letzte Beispiel deutet bereits an, dass auch die deutschsprachige Literatur keineswegs ausschließlich deutsch ist - und der vorliegende Aufsatz beschränkt sich aus heuristischen Gründen auf Texte, in denen das Deutsche eine der beteiligten Sprachen ist, wenngleich nur einer von ihnen (W. G. Sebalds Austerlitz) üblicherweise der deutschen Literatur zugerechnet wird.

Mehrsprachigkeit kann in literarischen Texten in verschiedensten Formen auftreten. Legt man vorläufig die in der Narratologie vorausgesetzte Unterscheidung zwischen discours und histoire zugrunde, so lassen sich diese Formen der Mehrsprachigkeit lokalisieren: auf der Ebene der histoire (wenn Figuren in der erzählten Welt verschiedene Sprachen sprechen), auf der Ebene des discours (wenn Texte mehrsprachig sind), oder im Verhältnis zwischen histoire und discours (wenn Figuren in der erzählten Welt eine andere Sprache sprechen als die des erzählenden Textes); und natürlich sind die verschiedensten Kombinationen solcher Mehr-als-ein-Sprachigkeit denkbar. Weil zur Unterscheidung solcher Verfahren noch keine eingebürgerte Terminologie vorliegt, sei hier, in heuristischer Absicht, ein kleines Kategorieninventar vorgeschlagen. ${ }^{1}$ Dabei werden im ersten Schritt einige Verfahren klassifiziert, um die Möglichkeit dieser Klassifikation in einem zweiten Schritt auch schon wieder in Frage zu stellen.

Die Wiedergabe eines Sprechens in einer fingierten Welt, die das in der histoire gesprochene Idiom im discours darstellt, sei (1.) glottamimetisch genannt. W. G. Sebalds Austerlitz besteht weitgehend aus wohlgeformten, hypotaktischen

1 Dabei werden neogriechische Komposita bevorzugt, um auf platonische und aristotelische Basisunterscheidungen zurückgreifen zu können, aber auch aufgrund einer gewissen Verehrung für Charles S. Peirce und Gérard Genette. Beiden Autoren war bzw. ist der heuristische Charakter der von ihnen vorgeschlagenen Kategorien weit stärker bewusst als den meisten ihrer Nachfolger in den Bereichen von Semiotik bzw. Narratologie, welche diese Kategorien in klassifikatorischem Interesse verwenden, statt an ihnen weiter zu arbeiten. 
Reden des Titelhelden, die nahezu ausschließlich auf Deutsch notiert werden einmal aber heißt es:

So ist mir unvergeßlich geblieben, daß [Austerlitz] seine Erläuterungen des bei der Fabrikation der hohen Wartesaalspiegel angewendeten Verfahrens beschloß, indem er, im Gehen noch einmal an den mattschimmernden Flächen emporblickend, sich selber die Frage stellte, combien des ouvriers perirent, lors de la manufacture de tels miroirs, de malignes et funestes affectations a la suite de l'inhalation des vapeurs de mercure et de cyanide. (19)

Der Umfang solcher glottamimetischer Verfahren kann zwischen zwei Polen variieren, die hier (1 a) ikonische und (1 b) indexikalische Glottamimesis genannt seien. Ein Fall von ikonischer Glottamimesis läge vor, wenn der discours auf der histoire-Ebene (das fingierte Sprechen in der erzählten Welt) mit dem discours auf der discours-Ebene (dem in der Erzählung notierten Sprechen) im gesamten Signifikanten-Material deckungsgleich wäre; ein Fall von indexikalischer Glottamimesis läge dann vor, wenn nur ein kleines sprachliches Indiz auf der discoursEbene das auf der histoire-Ebene gesprochene Idiom anzeigen würde.

Das zitierte Beispiel einer partiellen Glottamimesis liegt zwischen diesen beiden Polen, da zwar immerhin ein längerer Satz, damit aber nur ein winziger Teil der gesamten Rede von Austerlitz im discours des Romans auf Französisch erscheint. Beispiele für Extremwerte sind nicht in Sebalds Roman auszumachen, aber in Anita Desais englischsprachigem Roman Baumgartner's Bombay, auf den noch zurückzukommen sein wird. In denjenigen Passagen des Romans, die in Berlin spielen, ruft einmal (1 a) ein Zigarettenverkäufer „,Zigarren, Zigaretten““ (23) - und so kurz diese zitierte Rede ist, kann sie, da sie die gesamte Rede des Zigarettenverkäufers im Roman bildet, als ikonische Glottamimesis bezeichnet werden. Sagt jedoch jemand in denselben Passagen „,Mutti [...], take the child away, why is he still up?““ (34), so handelt es sich um (1 b) indexikalische Glottamimesis, insofern das eine Wort Mutti offensichtlich anzeigen soll, dass auf der histoire-Ebene der ganze Satz auf Deutsch geäußert worden sei. Indexikalische Glottamimesis wird häufig dort eingesetzt, wenn nicht vorausgesetzt werden soll, dass der Leser mehr als wenige Wörter der damit angezeigten Sprache spricht. ${ }^{2}$ Außer mit morphologischen Elementen lässt sich Anderssprachigkeit

2 Die Gruppe dieser Verfahren weist eine große Schnittmenge mit denjenigen auf, die Brigitte Rath ,Pseudoübersetzung“ nennt, also Texten, in denen „eine Äußerung einer Sprache eine Äußerung in einer anderen Sprache als deren imaginiertes Original evoziert, diese anderssprachige Äußerung aber nur durch eben diese Imagination zugänglich ist“ (16) - wobei für die Fälle indexikalischer Glottamimesis jedoch ein konstitutives fast vor dem nur einzufügen ist, da die Anderssprachigkeit dieser Äußerung eben nicht ausschließlich durch die Imagination, sondern durch - sei es auch nur wenige und kleine - markierbare Textelemente zugänglich ist. 
auch syntaktisch anzeigen, etwa mit einem Satz wie „,And [...] no lunch even have we had““ (136) - wobei freilich bestenfalls im Kotext darüber zu entscheiden ist, ob der Sprecher dieses Satzes ein nicht-idiomatisches Englisch spricht, das hier in ikonischer Glottamimesis abgebildet wird, oder ob es sich eben um eine indexikalisch glottamimetische Abbildung eines auf Deutsch gesprochenen Satzes handelt.

Warum jedoch lässt sich im Falle von Austerlitz überhaupt der eine Satz, den die Titelfigur auf Französisch spricht, als nur partielle Glottamimesis erkennen? Bei der Lektüre des zitierten Satzes, ja bei der Lektüre des Romans bis zu diesem Satz und darüber hinaus, könnte man auch annehmen, dass Austerlitz nur hier, wo er sich selbst eine Frage stellt, ins Französische fällt, während er doch sonst, mit dem Erzähler, Deutsch spricht. Später jedoch gibt der Erzähler ausdrücklich an, in welchen Sprachen seine Gespräche mit Austerlitz stattgefunden haben:

Da es mit Austerlitz so gut wie unmöglich war, von sich selber beziehungsweise über seine Person zu reden, und da also keiner vom anderen wußte, woher er stammte, hatten wir uns seit unserem ersten Antwerpener Gespräch stets nur der französischen Sprache bedient, ich mit schandhafter Unbeholfenheit, Austerlitz hingegen auf eine so formvollendete Weise, dass ich ihn lang für einen Franzosen hielt. Es berührte mich damals sehr seltsam, als wir in das für mich praktikablere Englisch überwechselten, daß nun an ihm eine mir bis dahin ganz verborgen gebliebene Unsicherheit zum Vorschein kam, die sich in einem leichten Sprachfehler äußerte und in gelegentlichen Stotteranfällen, bei denen er das abgewetzte Brillenfutteral, das er stets in der linken Hand hielt, so fest umklammerte, daß man das Weiße sehen konnte unter der Haut seiner Knöchel. (46)

Dies ist, mit der hier zugrunde gelegten platonischen Basis-Unterscheidung, ein Fall von (2) Glottadiegesis: ${ }^{3}$ Es wird erzählt, dass der discours auf der histoireEbene in einer bestimmten Sprache statthat, die aber als solche nicht notwendigerweise auch auf der discours-Ebene nachgebildet werden muss. Austerlitz enthält zwar einige fremdsprachige Elemente, darunter jedoch hauptsächlich Zitate aus fremdsprachigen literarischen Texten im Originalwortlaut (z.B. 38 u. 54), jedoch nur verhältnismäßig wenige glottamimetische Passagen, die ihrerseits interessante Komplikationen aufweisen.

Einmal erzählt Austerlitz, wie ihm als Kind ein Zettel mit dem Namen gegeben wurde, den er annehmen sollte: „Penrith-Smith hatte den Namen auf einen Zettel geschrieben, und als er diesen Zettel mir aushändigte, da wußte ich nichts anderes zu ihm zu sagen als ,Thank you, Sir‘, sagte Austerlitz.“ (98) Wenn

3 Die peirce'sche Terminologie ließe an dieser Systemstelle ,(1 c) symbolische Glottamimesis“ erwarten; das symbol im peirce'schen Sinne ist aber eben gerade nicht mimetisch, weshalb hier Peirce möge es mir verzeihen - die Triade in eine Dyade (Mimesis/Diegesis) mit einer Sub-Dyade (ikonische/indexikalische Mimesis) überführt wird. 
Austerlitz jedoch zum Zeitpunkt dieses Berichts ohnehin, wie der Erzähler ausgewiesen hatte, Englisch spricht, so ist der sprachliche Unterschied zwischen seiner berichtenden und seiner berichteten Rede vom Erzähler konstruiert, der nur die erstere Rede übersetzt, die letztere unübersetzt lässt. Offenbar ist Sebald hier die Unterscheidung zwischen der erinnernden Rede des erwachsenen und der erinnerten Rede des kindlichen Austerlitz wichtiger als eine glottamimetische Abbildung von beiden Reden.

Und in einem anderen Fall muss der Erzähler ausdrücklich, also glottadiegetisch, betonen, dass Austerlitz' Sprechen auf Deutsch ausnahms- und überraschenderweise, wenngleich nur hinsichtlich einiger Wörter, glottamimetisch zu verstehen ist, nämlich dort, wo er von seiner Lektüre einer deutschsprachigen Studie über das Ghetto von Theresienstadt berichtet:

Die Lektüre, die mir Zeile für Zeile Einblicke eröffnete in das, was ich mir bei meinem Besuch in der Festungsstadt aus meiner so gut wie vollkommenen Unwissenheit heraus nicht hatte vorstellen können, ging aufgrund meiner mangelhaften Deutschkenntnisse unendlich langsam vonstatten, ja, sagte Austerlitz, ich könnte wohl sagen, sie war für mich beinahe so schwierig wie das Entziffern einer ägyptischen oder babylonischen Keil- oder Zeichenschrift. Silbenweise mußte ich die in meinem Lexikon nicht aufgeführten, vielfach zusammengesetzten Komposita enträtseln, die von der in Theresienstadt alles beherrschenden Fach- und Verwaltungssprache der Deutschen offenbar fortlaufend hervorgebracht wurden. Und wenn ich die Bedeutung von Bezeichnungen und Begriffen wie Barackenbestandteillager, Zusatzkostenberechnungsschein, Bagatellreparaturwerkstätte, Menagetransportkolonnen, Küchenbeschwerdeorgane, Reinlichkeitsreihenuntersuchung oder Entwesungsübersiedlung Austerlitz artikulierte diese deutschen Schachtelwörter zu meiner Verwunderung ohne jedes Zögern und ohne die geringste Spur eines Akzents - endlich erschlossen hatte, so mußte ich, fuhr er fort, mit ebensolcher Anstrengung versuchen, den von mir rekonstruierten präsumtiven Sinn einzuordnen in die jeweiligen Sätze und in den weiteren Zusammenhang, der mir immer wieder zu entgleiten drohte, zum einen, weil ich nicht selten für eine einzige Seite bis nach Mitternacht brauchte und in solcher Zerdehntheit sich vieles verlor, zum anderen, weil das Ghettosystem in seiner gewissermaßen futuristischen Verformung des gesellschaftlichen Lebens für mich den Charakter des Irrealen behielt, obwohl es Adler ja beschreibt bis in das letzte Detail und in seiner ganzen Tatsächlichkeit. (334 sq.)

Mehrere Sprachen können jedoch sogar dann im Spiel sein, wenn sie weder als solche genannt werden noch in ihrer Signifikanten-Gestalt an der Oberfläche des Textes erscheinen. Ein Leser von Patrick Süskinds Roman Das Parfum wird etwa die naheliegende Vermutung hegen, dass die handelnden Figuren auf der histoire-Ebene, die nach Ausweis des allerersten Satzes in „Frankreich“ (5) spielt, Französisch gesprochen haben müssten. Der Text weist dies jedoch nicht ausdrücklich aus und flicht auch nur sehr wenige französische Signifikanten ein: Mehrfach wird jemand, immerhin bis hinein in die Schreibweise französisch, Maître genannt (98 et al.), einmal auch Monsieur (184). Ansonsten sprechen die 
Figuren, noch dort, wo ihre Rede zwischen Anführungsstriche gesetzt wird, ein unmarkiertes Deutsch. Falls also die Hypothese stimmt, dass in Süskinds Frankreich ,eigentlich' Französisch gesprochen werde, ist der Text durchgängig glottaadiegetisch und sehr weitgehend glotta-amimetisch.

Die Erzählung ist gleichwohl immerhin insofern glaubwürdig - im Rahmen von allgemein geteilten Annahmen über die sprachlichen Verhältnisse in der teils konstruierten, teils evozierten Welt der histoire -, als man annehmen kann, dass alle beteiligten Figuren in dieser Welt die gleiche Sprache sprechen und sich also untereinander verstehen. Dieser Sachverhalt sei hier glottapithanon genannt. ${ }^{4}$ Genauer: Insofern der Sachverhalt, dass sich die Figuren untereinander verstehen, nicht ausdrücklich motiviert wird - weil überdies anzunehmen ist, dass alle beteiligten Figuren in dieser Welt die gleiche Erstsprache sprechen - ist die Passage implizit glottapithanon. Wäre die Erstsprache einer der beteiligten Figuren eine andere Sprache als das Französische, so könnten wiederum verschiedene Fälle unterschieden werden: Würde dann etwa ausdrücklich erzählt, dass diese Figur das Französische gelernt hat, würde es sich um eine explizit glottapithanone Konstruktion handeln; wäre es jedoch kaum nachvollziehbar, warum sie Französisch sprechen kann und sie könnte sich trotzdem problemlos verständigen, müsste von einer glotta-apithanonen Konstellationen gesprochen werden.

Besondere Anforderungen an Glotta-Pithanisierung stellen fiktionale Erzählungen von first-contact-Begegnungen, also solchen, in denen die Figuren die Muttersprachen derjenigen, die sie treffen, schwerlich schon anderswo gelernt haben können; Erzählungen von Begegnungen mit Außerirdischen sind dafür schon seit Lukian, also keineswegs erst in der ,Science Fiction' im engeren Sinne - ein besonders beliebtes Experimentierfeld. ${ }^{5}$ Verstehen die Figuren einander, ohne dass der Leser versteht, warum sie sich verstehen, handelt es sich um eine glotta-apithanone Konstellation. Verstehen sie sich nicht, oder nur nach und nach mit einigen Mühen, ist die Konstellation glottapithanon. Wird ausdrücklich miterzählt, dass Außerirdische bereits eine Erdensprache - typischerweise diejenige des Textes, der von dieser Begegnung erzählt - gelernt haben oder wird in anderer Weise plausibilisiert, warum sich Außerirdische und Irdische verstehen, handelt es sich um eine explizite Glottapithanisierung. In Lukians „Wahrer Geschichte“ sprechen die Mondbewohner beispielsweise Griechisch, weil sie exilier-

4 Pithanón, ,glaubwürdig', etymologisch: ,zur Überredung geeignet‘, der Schlüsselbegriff der Rhetorik und Poetik (vgl. z. B. Aristoteles, 1461 b 12), wird hier anstelle von plausibel verwendet, um ein griechisch-lateinisches Hybrid-Kompositum zu vermeiden.

5 Entsprechende Texte sind dementsprechend ein besonders gut geeigneter Gegenstand zur Untersuchung von Verfahren der ,Pseudoübersetzung'; vgl. etwa Rath (15-20, zu C. S. Lewis, Out of the Silent Planet). 
te Griechen sind; in Voltaires „Micromégas“ lernt der Titelheld, ein knapp 40 km großer Sirius-Bewohner, binnen weniger Stunden Französisch durch das bloße Zuhören; sein winziger Reisegenosse vom Saturn, der kaum 2 km misst, schafft dies hingegen nur mit Mühen. Satirische oder parodistische Texte geben implizit $\mathrm{zu}$ verstehen, dass ihre expliziten Glotta-Pithanisierungen ihrerseits bewusst apithanon sind - das berühmteste Beispiel dafür ist der Babelfisch aus Douglas Adams' The Hitchhiker's Guide to the Galaxy, der, einmal in den Gehörgang eingeführt, es erlaubt, sämtliche in unserer Galaxie gesprochenen Sprachen zu verstehen. Und dieser Fisch spielt natürlich nicht nur, mit seinem Namen, auf die Babel-Episode der Genesis, sondern auch auf die ebenso berühmte Episode der epiphanischen Überwindung von Babel an, auf das Pfingstwunder aus der Apostelgeschichte.

Explizite oder implizite glottapithanone oder -apithanone Texte oder Textpassagen können in verschiedensten Kombinationen mit ikonisch oder indexikalisch glottamimetischen, glottadiegetischen, glotta-amimetischen oder glottaadiegetischen Texten oder Textpassagen vorkommen. Statt eine Matrix all dieser Kombinationsmöglichkeiten zu entwickeln, seien diese Kategorien in vier Lektüren erprobt, die wenigstens ansatzweise Texte in ihrer integralen Gestalt in den Blick nehmen. Erst danach wird die letzte im Untertitel dieses Beitrags versprochene Kategorie, das Glotta-Aporetische, eingeführt.

\section{Funktionen dieser Verfahren in der auchdeutschen Literatur}

Das kleine Korpus für die Sondierung besteht aus vier Texten, bei deren Beschreibung man auf die Frage ,Wie deutsch ist es?‘ (die den Titel eines dieser Texte in verunstalteter Form zitiert) die Antwort geben würde: ,eigentlich nicht, aber in mindestens einem wichtigen Punkt durchaus'. Die dominanten Sprachen dieser Texte sind das Italienische (Primo Levi, Se questo è un uomo, 1947), das Englische (Anita Desai, Baumgartner's Bombay, 1988; Walter Abish, How German Is It/Wie deutsch ist es, 1979) sowie das Spanische (Roberto Bolaño, 2666, 2004). All diese Texte wurden bereits ,ins Deutsche übersetzt', in allen Fällen jedoch waren die Übersetzer dabei mit schon übersetzten Elementen konfrontiert.

Komplementär zur Menge dieser Texte liegt die Menge derjenigen, bei deren Beschreibung man auf die Frage ,Wie deutsch ist es?' die Antwort geben würde: ,im Wesentlichen schon, aber in mindestens einem wichtigen Punkt nicht‘ (und dazu gehören etwa auch die meisten Texte von W. G. Sebald). Beide Textgruppen könnten als ,auch, aber nicht ausschließlich deutsche‘, abgekürzt ,auchdeut- 
sche‘, Literatur zusammengefasst werden. Während der Teilbereich der im Wesentlichen schon, aber in mindestens einem wichtigen Punkt nicht deutschen Literatur' in jüngster Zeit von der Germanistik intensiv erforscht wird, ${ }^{6}$ erscheint der Teilbereich der ,eigentlich nicht, aber in mindestens einem wichtigen Punkt durchaus deutschen Literatur' noch wenig erschlossen, da die ihm zuzurechnenden Texte üblicherweise verschiedenen anderen Nationalliteraturen zugerechnet werden. Die Kategorie der auchdeutschen Literatur und ihre beiden Unterkategorien stehen und fallen mit einem spezifischen Erkenntnisinteresse; es widerspräche daher nicht der Funktion dieser Kategorie, wenn bei näherem Hinsehen wenige Texte übrigblieben, die nurdeutsch oder garnichtdeutsch wären; ${ }^{7}$ die Kategorie eignet sich hingegen nicht als klassifikatorische, die etwa den Gegenstandsbereich einer Literaturgeschichte abzugrenzen erlauben würde.

(1. Bobelturm) Primo Levis unter dem Titel Ist das ein Mensch? erhältliches Buch erschien lange Zeit als eine bloße Übersetzung aus dem Italienischen und verschleierte dabei, wie deutsch schon das Buch Se questo è un uomo ist. Selbstverständlich war immer bekannt, dass sein Gegenstand, das Vernichtungslager von Auschwitz, ein Produkt von Deutschen ist; Levi führt aber auch vor, in welchem Maße die deutsche Sprache in diese Produktion involviert war. Dieser Sachverhalt wurde in einer Übersetzung neutralisiert, in der das Deutsche als bloße Zielsprache, nicht auch als Ausgangssprache, behandelt wurde, und wird erst in der Neuausgabe von 2011, welche die deutschsprachigen Elemente wenigstens teilweise markiert, etwas besser sichtbar. So erschien das Buch für den deutschen Leser weit harmloser als es ist. ${ }^{8}$

6 Wie die kaum noch überschaubare Forschung zu Autoren wie Emine Sevgi Özdamar, Yoko Tawada oder Feridun Zaimoglu belegt, hat sich die Germanistik in den letzten ungefähr zehn Jahren sehr intensiv Texten zugewendet, die sich als Kandidaten für diese Teilgruppe aufdrängen, insofern in ihnen jeweils mindestens eine andere Sprache deutliche Spuren hinterlassen hat. Bei diesen Texten ergibt sich dies überwiegend - denn Zaimoglu würde diese Beschreibung vermutlich zurückweisen - durch einen Eintritt ihrer Autoren ins Deutsche von außen (Stichwort: ,Migration'). Offensichtliche Mehrsprachigkeit kann aber auch aus anderen Sprachbewegungen resultieren. Zu denken wäre etwa auch an die Konfrontation deutschsprachiger Exilanten mit den Sprachen der Länder, in denen sie sich aufhalten (Stichwort: ,Exilliteratur'), oder aber daran, dass eine Sprache, wie derzeit vor allem das Englische, sich sogar unabhängig von menschlichen Bewegungen verbreiten kann und die Texte anderer Literaturen beeinflusst (Stichwort u. a.: ,PopLiteratur').

7 Man könnte sich, nebenbei bemerkt, wünschen, dass auch die scheinbar selbstverständlichen Kategorien der Nationalliteraturen nicht vorausgesetzt, sondern in Lektüren selbst erst begründet werden, dass also beispielsweise auch zur deutschen Literatur nur Texte gerechnet werden, bei deren Lektüre es sich als funktional erweist, sie eben zur deutschen Literatur zu rechnen.

8 Ähnliches gilt für Jonathan Littells Les bienveillantes; vgl. dazu: Koppenfels, 91-107. 
Zwar sind das Italienische und das Deutsche nicht die einzigen Sprachen in diesem Text. Levi beschreibt das Lager als riesiges Experiment auf die Frage des Menschseins, das „Tausende von Individuen, voneinander verschieden nach Alter, Stand, Herkunft, Sprache, Kultur und Sitten“ (ital. 145/dt. 108), zusammengebracht habe: „Nichts war ,kosmopolitischer“ als die Konzentrations- und Vernichtungslager der Nazis.“ (Levy/Sznaider 31) Entsprechend groß war die Menge der im Lager gesprochenen Sprachen: Levi schätzt sie auf fünfzehn bis zwanzig (itl. 124/dt. 90), unter denen er eine nicht identifizieren kann, „,he non sembra di questo mondo“ (ital. 36/dt. 27); bei der Ausgabe des Brotes warten die Häftlinge unter anderem auf „Brot-Broit-chleb-pane-pain-lechem-kenyer“ (ital. 63/dt. 46).

Unter all diesen Sprachen, von denen Levi glottadiegetisch berichtet, und von denen er einige in indexikalischer Glottamimesis aufruft, dominieren in seinem Texte deren zwei: das Italienisch seines Berichtes und das Deutsch der KZ-Erbauer, das wiederum den Bericht prägt. Den Karbidturm in Buna, errichtet mit „Ziegel, mattoni, briques, tegula, cegli, kamenny, bricks, téglak“, nennen die Häftlinge, nach Levis eigenem Bericht, nicht ihrerseits mit fünfzehn bis zwanzig verschiedenen Wörtern, sondern mit wenig mehr als einem Wort: „Babelturm, Bobelturm“ (ital. 24/dt. 90). Der Eigenname von Babel wird nur minimal dekliniert, wobei das Kompositum durch den zweiten Teil (-turm) auch in der Variante als ,deutsches' ${ }^{6}$ Wort lesbar bleibt. ${ }^{9}$ Das polyglotte Experiment wird von einem monoglotten Experimentator durchgeführt, der seine Sprache auch den Häftlingen aufzwingt oder ihnen allenfalls noch Raum für eine minimale Vokalverschiebung lässt.

Der mit Abstand größte Anteil von Levis indexikalischer Glottamimesis gilt der Terminologie der Täter; hätte der Übersetzer die schon im Original deutschsprachigen Elemente mit Sternchen „beleuchtet“10 - wie es sich erst seit ungefähr dreißig Jahren, vor allem in Übersetzungen von philosophischen Texten aus dem Französischen ins Deutsche, eingebürgert hat -, sähe eine Passage des Textes etwa so aus:

Das Resultat aus diesem erbarmungslosen, natürlichen Selektionsprozeß hätte man den Statistiken der *Lager entnehmen können. 1944 lebten in Auschwitz von den alten jüdischen

\footnotetext{
9 Bobelturm ist vermutlich - Levi kommentiert es ebensowenig wie der Herausgeber der annotierten Ausgabe, und google vervielfältigt nur einige hundertmal dieses hapax legomenonweniger als jiddisches Wort denn vielmehr als vom Jiddischen beeinflusste Aussprache eines deutschen Wortes zu verstehen; als sephardischer Jude muss Levi das Jiddische, das den aschkenasischen Juden im Lager als Verkehrssprache dient, erst im Lager lernen.

10 „Asteriscus adponitur in his quae omissa sunt, ut inlucescant per eam notam, quae deesse videntur.“ (Isidor, I. xx. 2); vgl. Stockhammer, Grammatik, 414-421 (auch zur weiteren Geschichte des Asteriskus).
} 


\begin{abstract}
Häftlingen (von den übrigen Häftlingen spreche ich hier nicht, denn ihre Bedingungen waren andere), von den *,kleinen Nummern' unter Hundertfünfzigtausend nur noch ein paar hundert; keiner von diesen war ein gewöhnlicher *Häftling in einem gewöhnlichen *Kommando und mit gewöhnlicher Ration. Es blieben nur die Ärzte übrig, die Schneider, Flickschuster, Musiker und Köche, attraktive junge Homosexuelle und Freunde oder Landsleute irgendwelcher Lagerautoritäten; darüber hinaus besonders rücksichtslose, kräftige und unmenschliche Individuen, die sich (vom Kommando der *SS dazu ausersehen, das in dieser Wahl eine satanische Menschenkenntnis an den Tag legte) als *Kapos, *Blockälteste und noch in anderen Ämtern behaupteten. (ital. 149/dt. 111) ${ }^{11}$
\end{abstract}

Mit der Notation von Inschriften reicht der Text in den Bereich ikonischer Glottamimesis hinein; in der folgenden Passage etwa sehen sogar Leser, die des Italienischen nicht mächtig sind, dass die Inschrift über dem Tor des Lagers „grell beleuchtet“ ist:

Il viaggio non durò che una ventina di minuti. Poi l'autocarro si è fermato, e si è vista una grande porta, e sopra una scritta vivamente illuminata (il suo ricordo ancora mi percuote nei sogni): ARBEIT MACHT FREI, il lavoro rende liberi. (ital. 34/dt.24) $)^{12}$

Unter diesen deutschsprachigen Elementen befinden sich auch weniger gebräuchliche, darunter sogar solche, über die man - jedenfalls solange sie im Text von einer anderen Sprache aufgerufen werden - durchaus lachen darf: „il misterioso burocrate tedesco che sovrintende a queste cose ci ha autorizzato alla erezione di uno ,Zweiplatziges Kommandoscheisshaus““ (ital. 223/dt. 170)

Hinzu kommt jedoch ein ganz anderes Feld deutscher Wörter. Levi hatte bereits vor seiner Gefangennahme Chemie studiert und kannte, weil das Deutsche vor dem Zweiten Weltkrieg sogar in einigen Naturwissenschaften noch als internationale Sprache diente, auch die entsprechende deutschsprachige Terminologie. Deshalb wurde er zur Arbeit im Chemielabor eingeteilt, und wahrscheinlich nur deshalb hat er das Vernichtungslager überlebt. Die im Originaltext deutsch, ohne Übersetzung, auftretenden Wörter „Kohlenwasserstoffe, Massenwirkungsgesetz“ (ital. 173/dt. 131) indizieren daher auch dieses, so unwahrscheinliche,

11 Asteriske von mir (R.St.) eingefügt; die neubearbeitete Übersetzung markiert zwar drei der deutschsprachigen Ausdrücke durch Kursivierung (kleine Nummern, Häftling, Blockälteste), lässt aber einige andere unmarkiert, darunter diejenigen, welche in der Übersetzung zu Komposita werden (statistiche del movimento dei Lager: Lagerstatistiken; comando delle SS: SS-Kommandos) und die hier in die Genitivkonstruktionen des Originals zurückaufgelöst werden, um auch deren deutschsprachige Anteile markieren zu können. Überdies ist das Mittel der Kursivierung uneindeutig, da Levi selbst es nicht für die Markierung einer anderen Sprache, sondern zur Hervorhebung verwendet, was die deutsche Übersetzung nachbildet (daher oben keiner für nessuno).

12 Vgl. a. die Inschriften im Waschraum: ,,Eine Laus, dein Tod““ und ,,Nach dem Abort, vor dem Essen / Hände waschen, nicht vergessen““ (ital. 65/dt.47). 
Überleben. $\mathrm{Zu}$ dessen notwendigen (selbstverständlich nicht hinreichenden) Voraussetzungen gehörte für Levi die zumindest partielle Mimikry an die Sprache der Täter, der Eintritt in die asymmetrische internationale Kommunikation, die sich aus der Vielsprachigkeit der Häftlinge und der Einsprachigkeit der Aufseher zusammensetzte.

Weil dieses Überleben zugleich die Bedingung dafür war, dass der Bericht überhaupt geschrieben werden konnte, erzählt dieser nicht nur von dieser Notwendigkeit der Mimikry, sondern nimmt sie in seine eigene Faktur auf. Ein Kapitel von Se questo è un uomo ist, wiederum im Originaltext deutsch, ohne Übersetzung, „Die drei Leute vom Labor“ überschrieben (ital. 221/dt. 168). Dieser Titel wird zwar in diesem Kapitel als Ausdruck des Kapo erklärt, der damit Levi und die beiden anderen Arbeiter im Chemielabor jeden Morgen abkommandiert (ital. 228/ dt. 174) und wäre insofern ein typischer Fall von Glottamimesis (an der Grenze von der ikonischen zur indexikalischen). Als Kapiteltitel jedoch wird dieser Ausdruck davon entkoppelt, einen discours innerhalb der histoire zu zitieren, und gehört einer Schicht des Textes an, die allenfalls noch indirekt im Dienst einer histoire steht. Mit Uspenskij lässt sich dies als „Kontamination eines AutorenTextes [...] mit dem Text einer beliebigen Figur“ (42) beschreiben, für die auch Uspenskij selbst schon Beispiele des Umgangs mit verschiedenen Sprachen gibt (vgl. 56-61 zum Einsatz des Französischen im russischen Originaltext bei Tolstoj und Puschkin).

(2. Pure silber) Anita Desai wurde (mit einem großartigen Druckfehler auf dem Rückumschlag zumindest der 7. Auflage von Baumgartner's Bombay) vom Independent angeblich als „[o]ne of the best English novelists writing in English“ bezeichnet. Selbstverständlich lautete das Lob im Original (und so auch auf dem Rücken anderer Auflagen): „One of the best Indian novelists writing in English“. Der Produzent des Druckfehlers hatte sich geweigert, die Differenzierung zwischen Herkunft des Autors und Sprache des Textes vorzunehmen, die bei der Klassifikation von postkolonialer Literatur üblich ist, und sie durch die plausible Tautologie ersetzt, derzufolge jemand, der auf Englisch schreibt, ein englischer Schriftsteller ist. (Der Druckfehler wäre übrigens ein Anlass um zu fragen, warum etwa Ian McEwan, auf den die Bezeichnung sogar im Sinne der vorgesehenen Ausdifferenzierung von geographischer und linguistischer Zurechnung passen würde, selten als „one of the best English novelists writing in English“ bezeichnet wird.)

Desais 1988 erstmals erschienener Roman Baumgartner's Bombay, ein zweifellos der englischsprachigen Literatur zuzurechnender Text, enthält immerhin einige Hinweise darauf, dass er in einem Land spielt, in dem außer dem Englischen auch andere Sprachen gesprochen werden: Der in Indien lebende Titelheld ist - wie in glottadiegetischen Passagen ausgewiesen und mit einigen indexikalisch glottamimetischen Elementen angezeigt wird - unter anderem mit dem 
Hindi und dem Bengali konfrontiert (92 et al.). Dieses Merkmal teilt Desais Roman mit sehr vielen, wahrscheinlich den meisten narrativen Prosatexten aus dem Bereich postkolonialer Literatur, in deren Erforschung daher die Beschreibung von solchen Sprachmischungen am weitesten fortgeschritten ist. Seit einer bahnbrechenden Studie von Chantal Zabus zu englisch- und französischsprachigen Romanen aus Afrika hat sich dafür die Metapher des Palimpsestes eingebürgert. Unter dem dominanten ,europhonen“ Text dieser Romane liegt danach ein ,Ethno-text‘ (Zabus 133 et al.), ein im Igbo oder Wolof ,gedachter` Text, der in einzelnen morphologischen oder syntaktischen Elementen durchscheint: entweder in einzelnen Wörtern oder kurzen Sätzen aus diesen Sprachen, die häufig (in Klammern, Fußnoten oder angefügten Glossaren) in die jeweils dominante Sprache der Texte übersetzt werden, oder aber in Eigenheiten, die in der europäischen Sprache als nicht (oder noch nicht) idiomatisch erscheinen, sich jedoch als ,Relexifizierungen' (101-155), also als Wort-für-Wort-Übersetzungen aus den lokalen Sprachen interpretieren lassen.

Sehr viel dichter als Elemente aus dem Hindi oder Bengali werden in Desais Roman jedoch solche aus dem Deutschen aufgenommen. Diese Sprache kommt vor allem über den Titelhelden ins Spiel, einen Juden, der als sehr junger Mann vor den Nazis nach Indien floh und dort noch fast fünfzig Jahre später, noch immer als schlecht integrierter firanghi (Desai, 20) und in ärmlichen Verhältnissen, lebt. Die in der Gegenwart, ungefähr zum Zeitpunkt der Veröffentlichung des Romans, spielenden Passagen werden mit heterodiegetischen, aber stark auf den Protagonisten fokussierten Rückblenden durchsetzt. In den Passagen, die in der Gegenwart spielen, interveniert das Deutsche zunächst nur in kleinen Bruchstücken, etwa wenn Baumgartner mit seinen Katzen spricht. Die Passagen hingegen, die die Vergangenheit evozieren, sind sehr stark mit Deutsch durchwoben, was sich im Druckbild vor allem durch mehr als zehn Lieder abbildet, aus denen ganze Strophen in Blockzitaten angeführt werden: vor allem Kinderlieder, aber auch „Kennst Du das Land/wo die Zitronen blühn?“ (47) Während fast alle deutschen Wörter und Satzfetzen im Fließtext übersetzt werden, bleiben die meisten dieser Gedicht- bzw. Liedtexte unübersetzt; nur „Die Wacht am Rhein“ wird in einer englischen Übersetzung (und nur in dieser) zitiert (115). Das poetische Deutsch steht überwiegend für dasjenige des ,guten' Deutschland; von dieser Regel machen jedoch zwei Verse aus Hans Baumanns „Es zittern die morschen Knochen“ (in der Variante mit „Heute gehört uns Deutschland“ statt des offenbar ursprünglich geschriebenen „Heute, da hört uns Deutschland“, 116) sowie der erste Vers aus Hoffmann von Fallerslebens „Lied der Deutschen“ (114 et al.) eine markante Ausnahme.

In besonderer Dichte setzt Desai überdies deutsche Wörter bzw. Satzfragmente in einer Passage ein, die im Telegrammstil Kriegsereignisse der Jahre 1939/40 
notiert (113ff). In anderen Passagen wird, erwartungsgemäß, das gastronomische Wortfeld besonders ausgiebig bemüht („Blutwurst, Leberwurst, Bratwurst“, 5; „Kartoffelpuffer or Leberknödel““, 71; „Weissbrot, Schwarzbrot, Pumpernickel“, 74; „Wienerschnitzel [...] Kartoffelpuffer“, 120). Daneben sind es vor allem Kosewörter, die aus der Vergangenheit des selbstverständlich gesprochenen Deutsch in Berlin in die Zeit in Bombay ragen, als Fragmente eines „German to which he [Baumgartner] was no longer accustomed“ (95): „,Meine kleine Maus,“ ,Mein Häschen, „Liebchen...““ (3) hatte Baumgartners Mutter ihren Sohn, erst mündlich (33), dann, noch bis zum Februar 1941, in Briefen (vgl. 164) genannt; „Liebchen“ nennt dieser seine Katzen $(5,146)$; dazwischen, aber offenbar nur in den ersten Jahren in Indien, verwendete er das Wort auch einmal gegenüber Lotte, einer gleichaltrigen Frau (200), der einzigen Person, mit der er später, als sie nur noch eine vertraute Freundin ist, gelegentlich Deutsch spricht (150).

Die Bandbreite der Verfahren, über die das Deutsche in den Roman Eingang findet, ist denen postkolonialer Romane im Umgang mit Sprachen wie Yoruba oder Wolof so analog, dass man konsequenterweise vom Deutschen als Sprache des ,Ethno-textes ${ }^{6}$ in diesem Roman sprechen könnte (Döring 203): ${ }^{13}$ So wie der Leser in einem in Rhodesien spielenden Roman covo und derere isst (Dangarembga 8), so isst er bei Anita Desais im gleichen Jahr erschienenen Roman Blutwurst und Kartoffelpuffer - alle sind einem durchschnittlichen englischen Leser ungefähr gleich wenig bekannt, und alle schmecken ihm, ohne dass er wüsste, worum es sich dabei eigentlich handelt. Wenn es gewöhnungsbedürftig erscheint, das Deutsche ebenso wie das Shona (aus dem covo und derere offenbar stammen) als Sprache einer ,Ethnie‘ zu verstehen, könnte dies als Anlass für die Frage dienen, ob der Ausdruck ,Ethno-text“ vielleicht schon problematisch ist, wenn mit ihm das Shona oder das Wolof als Sprachen von Ethnien klassifiziert werden, das Französische und das Englische hingegen nicht. Möglicherweise wäre es angemessener, sich auf die Palimpsest-Metaphorik zu beschränken und die Ausdrücke für die Sprachen der scriptio inferior (der unteren Schicht: meistens eine Muttersprache der Figuren, in Romanen aus Afrika etwa Shona oder Wolof, bei Desai jedoch das Deutsche) sowie der scriptio superior (der oberen Schicht: die dominante Sprache des Romans, bei Desai wie in vielen postkolonialen Texten das Englische) zu unterscheiden, ohne diese Sprachen in verschiedene Kategorien (Sprachen von Ethnien vs. solche von Nicht-Ethnien) aufzuteilen.

Jedenfalls ist das Deutsche in Baumgartner's Bombay nicht - wie die Sprachen der scriptio inferior in vielen postkolonialen Texten, oder vielleicht eher: in

13 Ich verdanke Tobias Döring den mündlichen Hinweis auf diesen Roman, der in seinem Aufsatz sehr viel konziser und einlässlicher dargestellt wird als im vorliegenden Abschnitt. 
der dominanten Deutung dieser Texte - einfach die Sprache, in der sich deren Sprecher zu Hause fühlen, während Fremdheit erst mit dem Sprechen anderer Sprachen begänne. Wie spannungsgeladen Baumgartners Verhältnis zum Deutschen ist, wird offensichtlich, als er in seinem Stammcafé den deutschen Hippie Kurt kennenlernt, den er in seine Wohnung aufnimmt. Die beiden Deutschen unterhalten sich bemerkenswerterweise, auch auf der histoire-Ebene, überwiegend auf Englisch. Dies wird ex negativo zuerst damit markiert, dass sich Kurt einmal dabei ertappt, das Wort medicine in einem englischen Satz deutsch auszusprechen (142), dann auch damit, dass an genau einer Stelle eine Abweichung von der Glottamimesis ausdrücklich glottadiegetisch ausgewiesen wird: „,Come with me. It's not far,' [Baumgartner] growled, in German.“ (143) Im Umkehrschluss legt dies nahe, dass große Teile des Gesprächs ,tatsächlich`auf Englisch geführt werden, deren Wiedergabe also glottamimetisch ist. Dieser Befund mag spitzfindig anmuten, ist jedoch im Feld der Literatur mit europäischen scriptio superior-Sprachen und nicht-dominanten scriptio inferior-Sprachen überraschend, da sich Muttersprachler der letzteren Sprachen dort typischerweise in diesen unterhalten, die Wiedergabe ihres Gesprächs also über weite Strecken glotta-amimetisch ist.

Im Rahmen von Desais Roman erscheint es daher auch glottapithanon, mischsprachige Sätze wie „Ja, it was so“ (155) als ikonische Glottamimesis fingierter Mischsprachigkeit innerhalb der zitierten Einheit, nicht also als indexikalische Glottamimesis eines fingierten einsprachig-deutschen Sprechens zu deuten (demzufolge Baumgartner auf der histoire-Ebene nicht ,Ja, es war so“ gesagt hätte). Selbst semantisch redundante Verdopplungen in zwei Sprachen müssen dann nicht in allen Fällen als Übersetzungen durch die Autor-Instanz verstanden werden. Denn so scheint es sich zwar bei Fällen wie „,Hörst du? D’you hear?““ (114) zu verhalten; bei „,It was wonderful. Es war prima.““ (160) legt jedoch schon die Reihenfolge der Sprachen nahe, dass Baumgartner ,tatsächlich“ in zwei Sprachen ,das Gleiche‘ gesagt habe. Auch dies wäre für Sprachhandlungen von Figuren in postkolonialen Texten eher untypisch. Oder handelt es sich um einen Übergang in der Wahrnehmung der am Gespräch Beteiligten (mit Uspenskij 47): Der eine sagt etwas auf Deutsch, was der andere auf Englisch ,versteht‘? Oder ,denkt‘ Baumgartner etwas auf Deutsch, was er auf Englisch sagt? Vielleicht sind aber all diese Versuche, einen zweisprachigen discours auf irgendeine kohärente zweisprachige Situation in der histoire zurückzubeziehen, hoffnungslos naiv, weil diese zweisprachigen Sätze „nicht unbedingt durch die Tendenz zur authentischen Wiedergabe in der Vorstellung des Autors motiviert, sondern ein Resultat spezieller kompositionstechnischer Aufgaben sind.“(Uspenskij 60)

Noch im gleichen Gespräch erreicht die Mischsprachigkeit eine weitere Stufe, an der die typographische Markierung der Sprachen scheitert. Der Hippie fragt 
nach dem Material der Pokale, die in Baumgartners Zimmer stehen: „,They are silber, man?““, und später, insistierend: „,Pure silber?““ (beides Desai 155) Nur Klein- und Recte-Schreibung unterscheiden ein deutsch ausgesprochenes englisches silber noch vom deutschen Silber; das Mittel der Kursivierung hingegen übernimmt plötzlich die neue Funktion, eine Betonung im Gesprochenen zu markieren. Die Sprachgestalt dieser Frage nach der Reinheit des Silbers ist auffallend unrein. Als Kurt von extremen Erfahrungen mit den verschiedensten religiösen Praktiken und verschiedensten Drogen in Indien, Nepal und Tibet erzählt, also an dem Tag, bevor er Baumgartner ermordet, ,bricht er ins Deutsche‘: „,Oh, I am krank - furchtbar krank, he broke into German.“ (156)

(3. En Voralmstrasse) Roberto Bolaños Roman 2666 bringt Personen aus vielen verschiedenen Ländern zusammen, und damit solche, die zwar nicht identisch viele, jedenfalls aber viele verschiedene Muttersprachen sprechen, und die aus den verschiedensten Gründen - als Literaturwissenschaftler, Reporter oder um den inhaftierten Sohn bekümmerte Mutter - miteinander kommunizieren. Vor allem im ersten, dritten und fünften Teil des Romans verdichten sich Begegnungen zwischen Personen verschiedener Muttersprachen, und der Erzähler nennt sehr häufig ausdrücklich die Sprache, in der die Gespräche stattfinden. Für den US-amerikanischen Sportreporter Fate dolmetscht (im dritten Teil) typischerweise eine Rosa das Spanisch einer anderen Rosa ins Englische; für Klaus Haas' Mutter Lotte dolmetscht (im fünften Teil) lange eine Ingrid, bis Lotte dank einiger Mühen und ihrer häufigen Mexiko-Reisen selbst in der Lage ist, mit der Rechtsanwältin ihres Sohnes Spanisch zu sprechen.

Überdies bringt 2666 eine Vielzahl von fingierten Intertexten ins Spiel, unter denen fingiert deutschsprachige überwiegen. Schon der erste Satz des Romans handelt von der Begegnung eines Lesers, Jean-Claude Pelletier, mit einem Buch des Schriftstellers Benno von Archimboldi, das aus der Sicht dieses Lesers in einer Fremdsprache abgefasst ist; schon auf der zweiten Seite reist Pelletier nach München, wo er in einer kleinen Buchhandlung in der Voralmstrasse ein weiteres Buch dieses Schriftstellers auftreibt. In der Folgezeit lernt er einige Archimboldi-Forscher aus anderen Ländern kennen, die sich bei ihren Begegnungen, wie glottadiegetisch markiert wird, in wechselnden Sprachen unterhalten. Von Archimboldi selbst wird später berichtet, er habe seinerseits (noch unter seinem ursprünglichen Namen Hans Reiter) Aufzeichnungen eines Boris Abramowitsch Anskys aufgefunden, die dieser offensichtlich auf Deutsch notiert hatte. Aus diesen Aufzeichnungen wiederum, die nicht ,zitiert‘, sondern ,referiert‘ werden, geht hervor, dass schon Ansky an Problemen der Fremd- und Vielsprachigkeit interessiert gewesen sein muss: Hat er doch seinerseits einen Roman geschrieben, in dem sich der Protagonist mit einem algenförmigen Außerirdischen, in einem teilweise unverständlichen Gespräch, über fremde Sprachen unterhält (sp. 900/dt. 949). 
Der Roman ist also hochgradig glottadiegetisch, oder genauer: poly-glottadiegetisch. Wenn er gleichwohl nicht eindeutig zur auchdeutschen Literatur zu rechnen ist, so weil er bemerkenswert wenig glottamimetisch ist. Und zwar ist er nicht nur nicht ikonisch glottamimetisch, sondern auch kaum indexikalisch glottamimetisch, enthält also nur sehr wenige deutschsprachige Elemente. Zwar sagt selbstverständlich mal jemand (fast) „Heil Hitler“ (sp. 948/dt. 1001), und einmal wird Heidegger - auf den zurückzukommen sein wird - mit dem Satz „Es gibt Zeit“ (sp. 244/dt. 254) zitiert. Aber sogar an dem sex appeal von Dienstgraden „de la Werhmacht“(sp. 837 [sic]/dt. 883) und „de la Luftwaffe“ (sp. 838/884), den etwa Jonathan Littell auskostet (Koppenfels 92), zeigt sich Bolaño auffallend desinteressiert. Schlimmer noch: Er bibliographiert überaus schlampig. Nach seiner Behauptung treibt etwa Pelletier in der Münchner Buchhandlung Archimboldos Büchlein El tesoro de Mitzi (sp. 16) auf - eine Behauptung, die erst von der deutschen Übersetzung richtig gestellt wird, da das Buch ja im Original den Titel Mitzis Schatz (dt. 12) tragen muss, insofern es kein Indiz dafür gibt, dass der Franzose ausgerechnet in Deutschland eine spanische Übersetzung dieses deutschen Buches aufgetrieben haben soll. Auch enthält 2666 nicht einmal in den Passagen, in denen auf der histoire-Ebene Deutsch gesprochen wird, Relexifizierungen, also keine Elemente indexikalischer Glottamimesis auf syntaktischer Ebene, auf die sich die Übersetzung ins Deutsche stützen könnten. ${ }^{14}$

Überdies ist der Roman nicht uneingeschränkt glottapithanon. Zwar wird immerhin stets motiviert, warum eine Figur eine andere Sprache als ihre Muttersprache beherrscht, und oft genug auch betont, wenn eine Figur eine bestimmte Sprache nicht beherrscht. Aber diese Beschreibungen sind weniger plausibel, als sie es sein könnten, wenn der Autor es ausdrücklich darauf angelegt hätte. Denn die Figuren können diese Sprachen, wenn sie sie einmal können, sozusagen zu gut. Wird einmal einer Figur die Kenntnis einer Fremdsprache zugeschrieben, so beherrscht sie diese, passiv wie aktiv, ohne Einschränkungen, wie ein Muttersprachler. Bolaño ist kaum daran interessiert, sich ein Sprechen in gebrochenen oder gemischten Sprachen auszumalen. Zwar wird gelegentlich von jemandem berichtet, er mische in seiner Rede spanische und englische Ausdrücke (sp. 493/ dt. 521); dieses ,Spanglish‘, für welches doch gerade das Gebiet an der Grenze von Mexiko zur USA berühmt ist, wird jedoch nicht glottamimetisch abgebildet - was umso überraschender ist, als man genau dies als sprachiges Komplement eines Romans erwarten könnte, der sich in vielen anderen Merkmalen so gut als ,Globalisierungsroman' beschreiben ließe.

14 Diese Auskunft verdanke ich Christian Hansen, dem solche Passagen als Übersetzer des Romans ins Deutsche am ehesten aufgefallen wären. 
(4. Echt Deutsch) Selbstverständlich kommt kein Buch über deutsche Geschichte zwischen 1933 und 1945 ohne den, sei es noch so minimalen, Einsatz von deutschen Wörtern aus; noch der Verfasser des Kolportageromans Stockhammer kennt nicht nur „Gruppenfuhrer“ (Kushel 46) und „Obersturmfuhrer“ (240), sondern will mit „mein eigenes kind“ sogar unter Beweis stellen, dass er korrekt deklinieren kann (331). Schlimmer noch ist, dass sich der Befund umkehren lässt: Fast in allen Texten, die zum Feld der ,eigentlich nicht, aber in einem wichtigen Punkt durchaus deutschen Literatur` nach $1945 \mathrm{zu}$ rechnen sind, wird diese Zugehörigkeit zumindest teilweise mit Ereignissen aus der Zeit zwischen 1933 und 1945 motiviert - entsprechend ambivalent ist die Beziehung der Sprecher zu dieser Sprache nicht nur bei Primo Levi, sondern auch bei Anita Desai.

Die Reflexion auf den Stand der deutschen Sprache nach dem Nationalsozialismus ist ein zentrales Thema von Walter Abishs 1979 erschienenem Roman How German Is It/Wie deutsch ist es. Die zweisprachig und ohne Fragezeichen gestellte Titelfrage kann sich auf den Inhalt oder die Sprachgestalt des Romans, vielleicht sogar auf die Illustration auf dem Titelblatt (einen barfüßigen Reiter mit kurzer Hose und Schirmmütze, dessen Pferd im Wasser steht) beziehen (Leucht 160). In einer kurzen Erzählung, aus der sich der Roman entwickelt hat, bezieht sich die Frage darauf, inwiefern Deutschland den Bildern entspricht, die man sich davon gemacht hat (Abish, The English Garden, 1 sq.) Der Roman, der diese erste Skizze mit einem dort noch kaum angelegten plot ausstattet, verhandelt hingegen vor allem die Frage, wie deutsch die deutsche Geschichte ist, wie sehr die Nachkriegsgeschichte von dem geprägt ist, was als die deutsche Geschichte verstanden wird. Denn Ulrich Hargenau, Sohn eines nach dem 20. Juli hingerichteten Mitglieds der Stauffenberg-Gruppe, gehörte in den 1970er Jahren zu den Gründungsmitgliedern der Terroristengruppe Einzieh und fragt sich, nachdem er sich von dieser wieder losgesagt hat, selbst: „Is it possible that I agreed to work with the group because I wanted a role that would, in a certain respect, parallel the role my father played in '44?" (251)

Damit hängt die Antwort auf die Frage zusammen, inwiefern sich deutsche Schriftsteller als „echt Deutsch“ charakterisieren lassen: „[Thomas Mann] remains echt Deutsch despite his dubious decision to abandon his country at its greatest time of need. Can anyone doubt or deny the significance of these writers [Mann und sechs andere zuvor aufgezählte]? Can anyone fail to recognize in these the attribute of a true German? Absolutely no irony intended.“ (124) Es ist nahezu unmöglich, den ausdrücklichen Ausschluss von Ironie nicht seinerseits ironisch zu lesen, zumal gerade ein englischsprachiger Schriftsteller bei „echt Deutsch“ schwerlich nicht an englisch-auchdeutsche Lyrik denken kann, an den 12. Vers aus T. S. Eliots The Waste Land: „Bin gar keine Russin, stamm’ aus Litauen, echt deutsch“ - ein Satz, der große Zweifel daran aufwirft, ob er stimmt, aber immer- 
hin, inmitten all der englischen Verse, unzweifelhaft ist, was er behauptet (,echt deutsch“). Wenn es trotzdem möglich wäre, Abishs Antworten nicht-ironisch zu verstehen, müsste aus ihnen wohl die These hervorgehen, echt deutsch sei der Widerstand gegen das echt Deutsche.

Dort jedoch, wo die Frage „How German is it?“ wörtlich (und diesmal mit Fragezeichen) im Roman gestellt wird, dort bezieht sich das it auf es selbst, auf German, „die deutsche Sprache“ (5, deutsch im Original - aber keineswegs alle deutschsprachigen Elemente im Roman werden kursiviert). Beantwortet wird die Frage aus der Perspektive des Philosophen Brumhold, der so berühmt ist, dass nach ihm sogar die Stadt Brumholdstein benannt ist, die ab 1962 an der Stelle erbaut wurde, an der zuvor das KZ Durst gestanden hatte (vgl. 80-82). Die dortige Bibliothek beherbergt unter anderem eine komplette Sammlung von Brumholds Büchern in ihrer „complete, revised 1974 edition“ (81), darunter „Die einzige Verführung, published in 1927“ (125), „Jetzt zum letzten Mal, and Ohne Grund, an early work published in 1936“ (49) - Abish bibliographiert seine erfundenen Intertexte wesentlich korrekter als Bolaño. Für Brumhold, „the German language remains the means and the key to [his] metaphysical quest [...] which is rooted in the dark soil of der Schwarzwald“, und er fragt sich, in einem Gedankenspiel des Erzählers: „How German is it still? Has is not [...] acquired foreign impurities, such as okay and jetlag and topless and supermarkt and weekend and sexshop, and consequently absorbed the signifiers of an overwhelmingly decadent concern with materialism?“ (5).

Abish, der in Wien geborene US-amerikanische Schriftsteller, spielt durch, wie das Englische aussähe, wenn es vom Deutschen annähernd so ,verunreinigt ${ }^{\text {‘ }}$ worden wäre wie dieses von jenem - und dieses philologische Experiment sprengt die philosophische Sorge um die Metaphysik. So wie Abish gar nicht erst versucht, die clichés über Deutschland zu korrigieren, sondern sie als solche vorführt, so sind auch die Wörter, die er dem Englischen zur Aufnahme empfiehlt, durchaus naheliegende Kandidaten: „die Autobahn“, „die Landschaft“, „der blaue Himmel“ (alle: 3), „Natur“, „Lederhosen“ (beide: 26), „Jägerschnitzel mit Pilzen“ (41), „Gründlichkeit“ (47), „Rumkugeln“ (149) oder „Dasein“ (169). Und Redewendungen wie: „Eigentlich ganz nett. Nicht wahr?“ (124) oder „Schönes Wetter, nicht wahr?“ (156). Abish tauscht nicht das Vertraute durch ein weniger Vertrautes aus, sondern macht es, als solches, unvertraut (Milich). Eine Lehrerin schreibt das Wort familiar an die Tafel (119), diskutiert es mit ihren Schülerinnen, und am Ende bleibt, wie der Roman in einem Satz registriert, der einen eigenen kurzen Absatz einnimmt, das Wort familiar an der Tafel stehen (122).

Allerdings steht an der Tafel nach Ausweis des Romans wohlgemerkt eben das Wort familiar, nicht etwa ein deutsches Äquivalent wie vertraut, obwohl es sich auf der Ebene der histoire um einen Deutsch-Unterricht in Brumholdstein 
handelt. Obwohl nahezu der gesamte Roman in Deutschland spielt, obwohl er so viele deutsche Wörter und Redewendungen enthält, ist er bemerkenswert wenig glottamimetisch. Es gibt keine glottapithanonen Indizien dafür, dass die Figuren auf deutschem Territorium - also mit Ausnahme einiger in Frankreich und der französischen Schweiz spielenden Passagen - eine andere Sprache als Deutsch sprechen könnten, und ziemlich zu Beginn des Romans wird dies, bei Ulrichs Einreise nach Deutschland, ausdrücklich glottadiegetisch markiert: „The passerby took it for granted that Ulrich spoke German.“ (12) Und dennoch sind die zahlreichen Dialoge des Romans zu mehr als 99\% auf Englisch zu lesen. Nur wenige der deutschsprachigen Elemente werden, wie etwa die Titel von Brumholds Büchern, als Zitate der im Text fingierten Welt motiviert. Sogar von dem gesprochenen Deutsch ist hingegen nur ein Teil, als ,direkte Rede', dem Sprechen spezifischer Romanfiguren zuzurechnen. Häufiger werden Redewendungen in Formulierungen wie die folgende eingebaut: „Sie haben einen schönen Hund, one of the men might say. “(31, Hvh. R.St.) Hier wird also nicht ein in der fingierten Welt gesprochener, sondern ein möglicher, in einem bestimmten Kontext typischerweise passender deutscher Satz ,zitiert'; Gegenstand ist nicht ein deutschsprachiger Sprecher, sondern das Deutsche.

\section{Das Glotta-Aporetische und andere Grenzen des Kategorieninventars}

Mehr noch: An mindestens einer Stelle fällt es sogar bemerkenswert schwer, das ,Pseudo-Original' des in der dargestellten Welt geführten Dialogs zu rekonstruieren. An dieser Stelle unterhalten sich Vin Kahnsitz-Lese und ihr Ehemann Albert über den bevorstehenden Besuch von Egon. Albert ergänzt: „He’s coming with a friend. A photographer.“ Vin aber fragt nach: „Male or female?“ (104) Da im Deutschen nicht leicht ein genusunmarkiertes Wort für friend und photographer zu finden ist, kann die Nachfrage im discours der histoire-Ebene ausgerechnet in diejenige Sprache schwer übersetzt werden, in der der Dialog nach der glottadiegetischen Fiktion stattfindet; ${ }^{15}$ die Wiedergabe kehrt die Logik der Relexifizierung eigentümlich um, da sie den Regeln der Sprache der scriptio superior, nicht denen der scriptio inferior entspricht. Insofern ist diese Szene nicht nur glotta-apithanon

15 Entsprechend ungeschickt ist - ohne dass ich eine geschicktere Lösung vorschlagen könnte die Übersetzung: „Er kommt mit jemandem, der fotografieren will. / Männlich oder weiblich? wollte Vin wissen.“ (Abish, Wie deutsch ist es, 135). Vgl. zu den spezifischen Übersetzungsproblemen bei diesem Roman: Leucht, 163 sq. 
(wie sie es etwa wäre, wenn das Ehepaar, unmotivierterweise, aber immerhin glottadiegetisch ausgewiesen, hier ,tatsächlich' miteinander Englisch spräche), sondern glotta-aporetisch: Es lässt sich kein konsistentes ,Original‘ der Rede ausmachen, die in der histoire gesprochen worden sein soll.

Etwas Ähnliches, wenngleich nicht ganz Strukturanaloges, geschieht in mindestens einem Detail von Bolaños 2666. Im dritten, im nördlichen Mexiko spielenden Teil fragt Fate in seinem Hotel in Santa Teresa nach einem Zugang zu einem Computer, bekommt aber von dem Mann an der Rezeption nur die Visitenkarte eines Internetcafés. Fate bittet nun den Mann, „que le tradujera el nombre del establecimiento. “ So verwunderlich schon diese Frage zum Text einer Visitenkarte ist, die ja üblicherweise als indexikalisches, nicht als symbolisches Zeichen behandelt wird, so ist die Antwort noch verwunderlicher: „El recepcionista se rió y le dijo que se llamaba Fuego, camina conmigo.“ Der Mann an der Rezeption übersetzt also, auf der Ebene des discours des discours, aus dem Spanischen ins Spanische, als habe Fate ihn gebeten, die Karte vorzulesen - tatsächlich bedeutet das Verb lamar ja eher ,heißen‘ als ,bedeuten‘, während doch ein Übersetzungsvorgang ein Verb wie bedeuten erwarten ließe (für die Angabe eines Signifikanten, der an die Stelle eines Signifikanten aus einer anderen Sprache tritt, wobei ein beiden gemeinsames Signifikat angenommen wird). Nicht zufällig ist die deutsche Übersetzung an dieser Stelle wiederum ,richtiger' als das spanische Original: „Der Mann lachte und sagte, der Name bedeute Feuer, geh mit mir.“ (alle Zitate: sp. 428/dt. 452, Hvh. R.St.) Auch in dieser Fassung jedoch ist nur ein Signifikant im Spiel (hier der deutsche), wo man zwei verschiedensprachige erwarten würde. Dass der Name des Internetcafés offenbar den Titel eines englischsprachigen Films zitiert, ${ }^{16}$ erleichtert die Rekonstruktion des Vorgangs in der histoire des Romans nicht.

Abish und Bolaño knüpfen mit solchen Momenten - wie bewusst, bleibe dahingestellt - an eine literarische Tradition an, zu deren berühmtesten Beispielen im Feld der vor-allem-aber-nicht-ausschließlich-deutschen Literatur Mignons ,Italien-Lied“ zählt, das zwar bei Anita Desai als ein typisch deutsches erscheint, dessen hypothetische ,Original'-Sprache aber aus dem Einführungszusammenhang in Wilhelm Meisters Lehrjahre nicht widerspruchsfrei rekonstruiert werden kann. ${ }^{17}$ Hebel spielt mit solchen glotta-aporetischen Momenten, wenn

16 Nämlich um David Lynchs Fire Walk With Me (1992), eine Art nachgelieferter Pilotfilm zur Twin Peaks-Serie; ich verdanke diesen Hinweis Hermann Doetsch.

17 Vgl. dazu (noch ohne den erst hier eingeführten Terminus): Stockhammer, Das Schon-Übersetzte, 285-290. Reinhard Babel hat überzeugend eine andere Variante des Glotta-Aporetischen in Novalis' Heinrich von Ofterdingen herausgearbeitet, wobei Novalis erkennbar auf die Szene der Einführung von Mignons Italien-Lied in den Lehrjahren anspielt (63-66). 
er seinen Zundelfrieder (auf Deutsch) behaupten lässt, er könne nur Polnisch sprechen - und die Schildwache an der deutsch-polnischen Grenze, die selbst nicht Polnisch spricht, ihm dies glaubt (Hamacher 14-17). Vielleicht jedoch ist dieses Glotta-Aporetische nicht nur ein halbwegs genau isolierbares Moment einzelner literarischer Texte, sondern ein Moment, an dem sich die Eigengesetzlichkeit der Literatur anzeigt: der Sachverhalt, dass sie weder mit realen noch mit imaginären Welten zu verrechnen ist.

Diese Hypothese sei kurz anhand eines der berühmtesten, wenngleich nicht meistgelesenen, Texte der auchdeutschen Literatur anskizziert, anhand von James Joyce' Finnegans Wake. Unter den - je nach Zählung „mindestens 16“ (Reichert 59), „rund vier Dutzend“ (Reichert 71) oder 65 (Deane xlviii) - Sprachen des Idioms, das Joyce für diesen und mit diesem Roman entwickelt, dürfte das Deutsche zu denjenigen gehören, welche die stärksten Spuren hinterlassen haben. Umso bemerkenswerter ist, wie wenig die hier vorgeschlagenen Kategorien zur Analyse von Finnegans Wake beitragen. Der Hauptgrund für dieses Scheitern ihrer Applikation liegt darin, dass der Roman die Unterscheidung zwischen histoire und discours ebenso unterläuft wie die Möglichkeit, ,direkte Rede‘ (also einen discours auf der histoire-Ebene) zu isolieren.

Wird etwa die Vielsprachigkeit noch des Ulysses zumindest an dessen Beginn mit der Glottamimesis von fingierten Einzelsprechakten motiviert - Buck Mulligan singt ,tatsächlich“ „Introibo ad altare dei“ -, so ist die Sprachigkeit des Finnegans Wake ganz in den discours transformiert. Zwar lässt sich Berichten von der Entstehung des Romans entnehmen, mit welcher Lust Joyce etwa in Zürcher Cafés Fragmente des Auchdeutschen sammelte; „,Really, it is not I who am writing this crazy book““, soll er gesagt haben, ,,It is you and you and that girl over there and the man in the corner." (Jolas 166) Ein Wort wie zurichschicken aber wird im Text nicht als Zitat einer Figurenrede motiviert (abgesehen davon, dass es wahrscheinlich ohnehin nicht einfach der Rede eines Zürchers abgelauscht wurde). Als Wake-Leser von durchschnittlicher Inkompetenz, der schon über die Auskunft staunt, dass der Roman immerhin einen nacherzählbaren plot haben soll, glaube ich zwar Sprach-Nester zu erkennen, Passagen mit überdurchschnittlich hohem Anteil identifizierbarer Subtextsprachen, die irgendwie mit Handlungselementen zusammenhängen: Das Wort zurichschicken etwa steht in einem Absatz, in dem ein „Herr Betreffender“ und „a Kommerzial“ vorkommen; irgendjemand schreibt hier „a reporterage on Der Fall Adams for the Frankofurto Siding“; und dass die Sprache dieser Passage „brockendootsch“ sei, lässt sich wohl sogar an der Syntax von Sprachgebilden wie dem folgenden erkennen: 
[...] and er, consstated that one had on him the Lynn O'Brien, a meltoned lammswolle, disturbed, and wider he might the same zurichschicken other he would, with tosend and obertosend tonnowatters, one monkey’s damages become. (alle Zitate: 69 sq.)

Es ist jedoch offenbar unmöglich, die Sprachelemente dieser Passage nach den jeweiligen Beiträgen von handelnden Figuren auszuwerten - so dass hier schwerlich eine glottamimetische Beziehung besteht.

Ebenso schwer ist dieser Roman in ein Kategorieninventar einzutragen, das Meir Sternberg zur Klassifikation von verschiedenen Formen der Sprachigkeit in der Literatur vorgeschlagen hat. Sternberg erzeugt dabei aus der Unterscheidung zwischen Ein- und Mehrsprachigkeit einerseits und der zwischen histoire- und discours-Ebene andererseits eine vierstellige Matrix (224), mit der sich neben einzelnen Verfahren auch die Tendenzen ganzer Texte beschreiben lassen: Entweder sind histoire- und discours-Ebene (bei Sternberg: object und medium) unilingual, oder beide polylingual, ${ }^{18}$ oder nur jeweils eine von beiden ist unisowie die jeweils andere polylingual. Sollte es, beispielweise, einen deutschsprachigen fiktionalen Text geben, in dem in der fingierten Welt ausschließlich Deutsch gesprochen wird und der ausschließlich auf Deutsch geschrieben ist (also mit „unilingual object“ und im „unilingual medium“), so würde dieser, mit Sternbergs Kategorien, zum Fall der (1.) „referential restriction“ zählen. Austerlitz hingegen (poly/poly) entspricht immerhin teilweise dem (2.) „vehicular matching“. Beides wären nach der hier vorgeschlagenen Unterscheidung Fälle von Glottamimesis. Romane wie Süskinds Parfum (poly/uni) hingegen folgt weitgehend der (3.) „homogenizing convention“, da das in der Welt der histoire gesprochene Französisch kaum Spuren im discours hinterlässt.

Die letzte Möglichkeit, (4.) „,vehicular promiscuity“, die nach allen Regeln der Kombinatorik die Variante uni/poly abbilden müsste, wird von Sternberg jedoch mit einem Doppelstrich von den anderen abgetrennt. Sie umfasst nämlich Texte, von der makkaronischen Poesie bis eben zu Finnegans Wake, bei denen sich kaum entscheiden lässt, ob das zugrundeliegende object dieses Schreibens tatsächlich, wie die Kombinatorik es erwarten ließe, unilingual ist; vielmehr notiert Sternberg zu dieser Klasse im Objektbereich: „variable, possibly unilingual“. Mehr noch: Sogar unter der Voraussetzung einer mehrsprachigen Welt der histoire-Ebene wäre Finnegans Wake nicht einfach in die (2.) Gruppe von Texten mit der Tendenz „vehicular matching“ $\mathrm{zu}$ rücken, die doch eigentlich systematisch für die Kombination polylingual object/polylingual medium vorgesehen ist. Denn Objekt und

18 Dieses griechisch-lateinische Hybrid-Kompositum ist damit zu rechtfertigen, dass es seine eigene Bedeutung verkörpert. 
Medium ,matchen“ (wie es neuerdings auchdeutsch heißt) dort nicht; Zürcher etwa sagen nicht zurichschicken. Wahrscheinlich ist die im Wake evozierte Welt polyglott, offensichtlich ist die Gestalt des Wake polyglott - und trotzdem besteht zwischen beiden keine glottamimetische Beziehung (,so every act of bilingual interference is not necessarily an instance of mimetic transposition“, 228).

Wenn Finnegans Wake einen Grenzwert des Literarischen überhaupt darstellt, so unter anderem insofern, als er mit der Konvention der ,direkten Rede‘ in der Literatur bricht und damit einen Anlass bietet, diese Konvention als solche in Frage zu stellen. Zwar scheinen sich, in den meisten anderen fiktionalen Texten, die Figurenreden so scharf von der Erzählerrede abgrenzen zu lassen wie Zitate vom ,eigenen' Text nach den Konventionen wissenschaftlichen Arbeitens, so dass beide Abgrenzungen die typographische Form ihrer Markierung (Anführungsstriche auf/Anführungsstriche zu) miteinander teilen. Doch ist diese Konvention für die Figurenrede fast nur in der deutschsprachigen Literatur, und auch in dieser nicht restlos durchgesetzt. Sebald etwa verzichtet durchgängig auf Anführungsstriche zur Markierung von Austerlitz' Rede, und zwar unabhängig davon, ob er diese als direkte (z.B. 14) oder als indirekte (z.B. 16) wiedergibt - was von einem zum anderen Satz wechseln kann. Im Englischen und Französischen werden ohnehin auch scheinbar eindeutige Fälle ,direkter Rede‘ zwar am Anfang, aber nicht immer ebenso deutlich auch am Ende typographisch markiert; bei dem im Französischen verbreiteten style indirect libre wäre es ganz unmöglich, logisch korrekte Anführungsstriche zu setzen.

Wird jedoch die trennscharfe Unterscheidung zwischen ,direkter Rede، (vorgeblich zitierter discours in der histoire) und Erzählerrede (discours des discours) in Frage gestellt, und lässt sich gar, wie im Falle der glotta-aporetischen Momente, eine ursprüngliche ,direkte Rede' gar nicht konsistent rekonstruieren, so bricht damit die Möglichkeit zusammen, überhaupt eine in sich selbständige histoire zu interpolieren, die vom discours unabhängig wäre. Nicht nur beruht eine solche Interpolation bei fiktionalen Texten ohnehin auf der metonymischen Vertauschung von Ursache und Wirkung: Während der discours vorgibt, eine histoire nur wiederzugeben, hat er diese erst produziert (Culler 183). Fraglich ist überdies, ob diese Interpolation immer möglich oder gar notwendig ist, ob also wirklich alle Sätze eines fiktionalen Textes als „presentation of events“ $\mathrm{zu}$ verstehen sind, wobei anzunehmen sei, „that these events in principle have features not reported by the discourse, such that the selection operated by the discourse has meaning“ - Eigenschaften, die etwa Jonathan Culler zufolge sogar für „the most radical fictions“ gelten sollen (alle Zitate: 187). Zur Debatte steht, anders gesagt, ob jeder Text eine ,Welt‘ entwirft, indem er sie mit einigen ausgewählten Prädikaten (186) skizziert, die dann - in der Imagination des Lesers - um weitere Prädikate ergänzt werden können, so dass diese ,Welt‘ eine zunehmend konkretere 
und vollständigere Gestalt annimmt. ${ }^{19}$ Nur für diesen Bereich der Imagination, die vielleicht von fiktionalen Texten inspiriert wird, aber nicht $z u$ diesen gehört, lässt sich dann etwa eine Frage wie diejenige beantworten, in welcher Sprache eine Figur in der histoire spricht, von der dies nicht ausdrücklich angegeben ist. Zwar regelt ein Konglomerat von Wissen über die außerliterarische Welt einerseits, über literarische Darstellungskonventionen andererseits, das Verhältnis von Fiktion und Imagination; im Rahmen von Annahmen, die teils auf dem common sense, teils auf akkumulierten Leseerfahrungen beruhen, erscheint es etwa als plausibel, dass in Süskinds Frankreich ,eigentlich` französisch gesprochen wird, oder Sherlock Holmes mit zwei Nasenflügel auskommen muss, obwohl der Leser über keine dieser Angaben verfügt. Da Literatur jedoch definitionsgemäß eben diese Darstellungskonventionen in Frage stellt, ist nicht auszuschließen, dass Sherlock Holmes vielleicht doch drei Nasenflügel besitzt, oder in Süskinds Frankreich eben Deutsch gesprochen wird.

Dementsprechend beruhen sehr viele Aussagen über den glotta-mimetischen oder glotta-amimetischen Charakter von Reden in literarischen Texten auf mehr oder weniger plausiblen Konjekturen. Glotta-aporetische Momente hingegen verunmöglichen solche Konjekturen systematisch, insofern aus ihnen auf keine in sich konsistente Sprach-Welt (in der histoire) zu schließen ist. Sie betonen besonders nachdrücklich, dass es letztlich nichts gibt als den discours, dass die in ihm hergestellte (Mehr-, Viel-, oder vielleicht auch Ein-) Sprachigkeit nicht nur keiner realen, sondern auch keiner fingierten Sprach-Welt gegenüber sich rechtfertigen muss. Insofern indizieren solche glotta-aporetischen Momente die Eigengesetzlichkeit des Literarischen.

Diese Zuspitzung wird schwerlich als Ergebnis jeder Analyse funktional sein, zumal wenn man daran interessiert ist, Literatur auf transkulturelle Beziehungen oder Globalisierungsprozesse zu beziehen und sich dabei bestimmte Konstellationen von Sprachen am anschaulichsten so vorstellt, dass sie durch personale Agenten verkörpert werden: Figuren, die nur eine Sprache sprechen; Figuren, die mehrere Sprachen sprechen; Figuren, die dolmetschen usw. Wahrscheinlich regiert dabei jedoch allzu selbstverständlich die Erwartung, die vielsprachige Welt müsse poly-glottamimetisch, im Modus eines „vehicular matching“ zwischen „polylingual object“ und „polylingual medium“ (Sternbergs 2. Gruppe), in vielsprachigen Texten abgebildet werden.

Möglicherweise entspricht diese Annahme jedoch einer naiven Sprachtheorie, die jedenfalls von auffallend vielen literarischen Texten nicht eins $\mathrm{zu}$ eins

19 Vgl. Ryan zu einem ausgeführten Modell dieser Vorstellung einer fiktiven Welt, die vom Text nur repräsentiert werde und von ihm partiell unabhängig sei. 
umgesetzt wird. Nicht nur können Romane, die in einer durchaus globalen, vielsprachigen Welt spielen, wie etwa Bolaños 2666, bemerkenswert einsprachig sein - und damit eher der „homogenizing convention“ (Sternbergs 3. Gruppe) folgen. Mehr noch: Sogar mehrsprachige Texte, die in der einen oder anderen Beziehung zu mehrsprachigen Welten stehen, folgen selten ausschließlich dem Prinzip des „vehicular matching“; fast immer interveniert „vehicular promiscuity“. Schon in Michail Bachtins Konzeption ist die Polyphonie des Romans nicht etwa einer zählbaren Menge von Stimmen zuzurechnen, von denen jede in einer ihr eigenen Sprache spricht, sondern entsteht aus Redeakten des Erzählers sowie der von ihm konstruierten Figuren, die voneinander nicht immer durch Anführungsstriche säuberlich getrennt werden können. ${ }^{20}$ Auch die Texte von Levi, Desai und Abish enthalten - bei allen offensichtlichen Unterschieden gegenüber dem Finnegans Wake - deutschsprachige Elemente in Schichten des Schreibens, die sich nicht der histoire-Welt zurechnen lassen (weder Redeakten der Figuren noch dort vorliegenden Inschriften bzw. Buchtiteln): ein Kapiteltitel bei Levi, die Lieder bei Desai, die möglichen und typischen, aber nicht in erzählten Gesprächen realisierten Redewendungen bei Abish. ${ }^{21}$ In all diesen Fällen übernimmt der discours die gesamte Verant-Wortung, ohne sich dafür auf eine fiktionale ,Welt ${ }^{*}$ berufen zu können, um deren mimetische Darstellung es zu tun wäre. Jeder literarische Text, nicht nur ein so überraschend fast-nur-spanischsprachiger wie 2666, sondern auch ein ,alle Sprachen in sich kontrahier[ender]“ (Hamacher 28) wie Finnegans Wake, ist insofern einsprachig: Jeder entwickelt das Idiom, in dem er verfasst ist. Vielleicht ist gerade dies - jenseits von glottamimetischen Abbildungen einer vielsprachigen globalisierten Welt - ein spezifisch literarischer Beitrag zur sprachlichen Dimension von Globalisierungsprozessen.

\section{Works Cited}

Abish, Walter. How German Is It/Wie deutsch ist es. 16th ed. New York: New Directions, o.J. --- . Wie deutsch ist es. Trans. Renate Hampke. Reinbek bei Hamburg: Rowohlt, 1996.

--- . „The English Garden.“ In the Future Perfect. London/Boston: Faber and Faber, 1984. 1-21.

20 Einmal vorausgesetzt, dass die unter dem Autornamen Vološinov veröffentlichte Studie tatsächlich - wie dies inzwischen die Mehrheitsmeinung ist - von Michail Bachtin stammt: So erklärt sich das besondere Interesse dieser Studie am style indirect libre damit, dass sich in diesem Modus exemplarisch Bachtins Konzept einer Redevielfalt ohne jeweils zurechenbare ,Stimmen realisiert. Vgl. dazu ausführlicher: Stockhammer, Grammatik, 352-362. Auch Uspenskij (46 et al.) knüpft ausdrücklich an Vološinov an.

21 Schon Eliots „echt deutsch“-Vers lässt sich ebenfalls nicht ohne weiteres einer erzählten Sprechsituation zurechnen. Vgl. die Details bei: Stockhammer, Das Schon-Übersetzte, 276 sq. 
Aristoteles. Poetik. Trans. and ed. Manfred Fuhrmann. Stuttgart: Reclam, 1982.

Babel, Reinhard. Translationsfiktionen. Zur Hermeneutik, Poetik und Ethik des Übersetzens. Ketsch bei Mannheim: Mikroform, 2014.

Bachtin, Michail. „Das Wort im Roman.“ Untersuchungen zur Poetik und Theorie des Romans. Trans. Michael Dewey. Berlin/Weimar: Aufbau, 1986. 77-261.

Bolaño, Roberto. 2666. Barcelona: Anagrama, 2004. (quoted as “sp.")

--- . 2666. Trans. Christian Hansen. Frankfurt a. M.: Fischer Taschenbuch, 2011. (quoted as "dt.")

Culler, Jonathan. „Story and Discourse in the Analysis of Narrative.“ The Pursuit of Signs: Semiotics, Literature, Deconstruction. Ithaca, NY: Cornell UP, 1981. 169-87.

Dangarembga, Tsitsi. Nervous Conditions. A Novel. Boulder/CO: Ayebia, 2004.

Deane, Seamus. „Introduction“. James Joyce, Finnegans Wake. 10th ed. London etc.: Penguin, 2000. vii-l [7-50].

Desai, Anita. Baumgartner's Bombay. London: Vintage, 2007.

Döring, Tobias. „A Meaning to the Meaningless: Anita Desai's ,Baumgartner’s Bombay“.“ Beyond ,Other Cultures': Transcultural Perspectives on Teaching the New Literatures in English. Ed. Sabine Doff and Frank Schulze-Engler. Trier: Wissenschaftlicher Verlag, 2011. 193-207. Hamacher, Werner. „Kontraduktionen.“ Transmission - Übersetzung, Übertragung, Vermittlung. Ed. Georg Mein. Wien: Turia \& Kant, 2010.13-34.

Isidor von Sevilla. Etymologiae sive Origines. Torino: Unione Tipografico-Editrice Torinese, 2004. Jolas, Eugene. Man from Babel. Ed. Andreas Kramer and Rainer Rumold. New Haven/London: Yale, 1998.

Joyce, James. Finnegans Wake. Ed. Seamus Deane. 10th ed. London etc. Penguin, 2000.

Koppenfels, Martin von. Schwarzer Peter. Jonathan Littell und die Identifikation mit dem Täter. Göttingen: Wallstein, 2012.

Kushel, Cash. Stockhammer. A Novel. Boca Raton, Florida: Rosscott \&Jordan, 2010.

Leucht, Robert. „Prozesse und Aporien der Übersetzung bei Walter Abish: Vier Fallstudien.“ Exilforschung: Ein internationales Jahrbuch. Ed. Claus-Dieter Krohn et al. München: edition text\&kritik, 25 (2007): 153-70.

Levi, Primo. Se questo è un uomo. Torino: Einaudi, 1989. (quoted as „ital.“)

--- . Ist das ein Mensch? Trans. Heinz Riedt. Ist das ein Mensch? - Die Atempause. München: Hanser, 2011. 7-219. (quoted as „dt.“)

Levy, Daniel, and Natan Sznaider. Erinnerung im globalen Zeitalter. Der Holocaust. 2001. Aktualisierte Neuauflage. Frankfurt a. M.: Suhrkamp, 2007.

Milich, Klaus J. „Lektüre der fremden Zeichen: Walter Abish's Literarisierung der Wahrnehmung im intertextuellen Diskurs mit der postmodernen Anthropologie."Amerikastudien/American Studies 38.2 (1993): 181-202.

Rath, Brigitte. „Unübersetzbares, schon übersetzt: Sprachliche Relativität und Pseudoübersetzungen. “Les intraduisibles/Unübersetzbarkeiten: Sprachen, Literaturen, Medien, Kulturen / Langues, littératures, médias, cultures. Ed. Jörg Dünne et al. Paris: Éd. des Archives contemporaines, 2013. 15-25.

Reichert, Klaus. Vielfacher Schriftsinn. Zu „Finnegans Wake“. Frankfurt a. M.: Suhrkamp, 1989. Ryan, Marie-Laure. Possible Worlds, Artificial Intelligence, and Narrative Theory. Bloomington: Indiana UP, 1991.

Sebald, W.G. Austerlitz. München: Süddeutsche Zeitung/Bibliothek, 2008.

Sternberg, Meir. „Polylingualism as Reality and Translation as Mimesis.“ Poetics Today 2 (1981): 221-39. 
Stockhammer, Robert. „Das Schon-Übersetzte: Auch eine Theorie der Weltliteratur.“ Poetica 41 (2009): 257-91.

--- . Grammatik. Wissen und Macht in der Geschichte einer sprachlichen Institution. Berlin: Suhrkamp, 2014.

Süskind, Patrick. Das Parfum. Die Geschichte eines Mörders. 1985. Zürich: Diogenes, 1994.

Uspenskij, Boris A. Poetik der Komposition. Ed. Karl Eimermacher. Trans. Georg Mayer. Frankfurt a. M.: Suhrkamp, 1975.

Vološinov, Valentin N. Marxismus und Sprachphilosophie. Grundlegende Probleme der soziologischen Methode. Trans. Renate Horlemann. Frankfurt a. M. etc.: Ullstein, 1975.

Zabus, Chantal. The African Palimpsest. Indigenization of Language in the West African Europhone Novel. Amsterdam/Atlanta, GA: Rodopi, 1991. 